\title{
Unités syntaxiques et unités intonatives majeures en français parlé : inclusion, fragmentation, chevauchement
}

\author{
Anne Lacheret-Dujour' et Sylvain Kahane \\ Modèles, Dynamiques, Corpus (MoDyCo) - https://www.modyco.fr/fr/ \\ CNRS : UMR7114, Université Paris Nanterre La Défense
}

\begin{abstract}
Résumé. Cet article, consacré à l'interface intonosyntaxique en français parlé, explore les relations entre les unités prosodiques et syntaxiques majeures, respectivement appelées périodes intonatives et unités illocutoires dans le modèle utilisé. Il présente différents types d'appariement entre les périodes intonatives et les unités illocutoires, et traite de trois constructions de base : l'alignement, lorsque les frontières prosodiques et syntaxiques coïncident ; l'inclusion, quand une période intonative regroupe plusieurs unités illocutoires ; et la fragmentation, lorsqu'une unité illocutoire est segmentée en plusieurs périodes intonatives. Est également étudié un cas singulier de désynchronisation, où ni les frontières prosodiques ni les frontières syntaxiques ne coïncident. Ces différentes constructions sont discutées à la lumière des contraintes communicationnelles et discursives.
\end{abstract}

\begin{abstract}
Major prosodic and syntactic units in spoken French: inclusion, fragmentation, overlapping This paper is devoted to the intonosyntactic interface in spoken French, by exploring relationships between major prosodic and syntactic units, respectively called intonational periods and illocutionary units in the developed model. It presents different kinds of matching between intonational periods and illocutionary units, and discusses three basic constructions: Alignment, when prosodic and syntactic boundaries coincide; inclusion, when an intonational period groups several illocutionary units; and fragmentation, when an illocutionary unit is segmented by several intonational periods. We also exhibit a singular case of desynchronization, when neither prosodic boundaries nor syntactic boundaries coincide with each other. These different patterns are discussed in the light of communicative and discourse constraints.
\end{abstract}

\section{Introduction}

C'est dans les années 1970 que le terme intonosyntaxe est introduit en France pour désigner l'étude des relations entre intonation et syntaxe (Di Cristo 1975). Si à l'époque on établit une corrélation directe entre l'agencement prosodique d'une phrase et sa structure 
syntaxique, cette hypothèse de congruence intonosyntaxique est rapidement mise en question, d'autres éléments jouant un rôle important dans la structuration prosodique du message. C'est ainsi que Rossi (1981) met en avant le rôle central de l'intonation dans l'encodage de la structure informationnelle pour expliquer cette congruence relative. Martin $(1981,1987)$ souligne quant à lui le conditionnement rythmique des phrasés intonatifs. On peut donc partir d'un premier constat: ni nécessairement congruente à la syntaxe, ni totalement indépendante, la structure intonative est associée à la syntaxe dans la mesure où d'une part elle participe à l'actualisation de la structure communicative dans le message, d'autre part, elle respecte également des principes d'équilibre et d'empan rythmique.

Aujourd'hui, les chercheurs s'accordent pour dire que si l'intonation n'est pas complètement autonome par rapport à la syntaxe c'est qu'elle doit répondre à deux contraintes syntaxiques majeures, formulées le plus généralement dans le cadre des grammaires de dépendance (Mertens 1993, 1997, 2008) : le principe de cohésion et le principe de connexité. Selon le premier, certains segments appelés chunks syntaxiques (Abney 1991) sont particulièrement cohésifs et ne peuvent pas être séparés par des frontières prosodiques. C'est le cas des clitiques préverbaux et de leurs têtes verbales (il le lui donne). D’une façon plus générale, le trait [ \pm clitique] doit être pris en compte pour la dérivation de la structure prosodique : une unité intonative complète contient au moins un mot non clitique. Quant au principe de connexité, nous le reformulons comme suit : deux chunks syntaxiques peuvent fusionner dans une unité intonative s'ils sont connexes (ex.1), c'est-à-dire s'il existe entre leurs têtes une relation de dépendance (ou connexion), s'ils ne le sont pas, ce regroupement est interdit (ex. 2).

(1) (cet exercice vraiment infernal) (a fait suer les étudiants de master)

(2) *(cet exercice) (vraiment infernal a fait suer) (les étudiants de master)

Deux problèmes majeurs restent néanmoins en suspens : (i) que faire et que dire quand les énoncés, nombreux en français parlé, ne sont pas marqués par des relations de dépendance explicites mais construits autour de la juxtaposition de segments qui constituent ainsi des îlots syntactico-sémantiques ${ }^{i}$ (Cresti et Moneglia 2018), (ii) si les contraintes morphosyntaxiques pèsent fortement dans le regroupement des unités de bas niveau, comment, au-delà des chunks, s'effectue la cohésion intonosyntaxique, sur quels types d'unités, définies selon quels critères ? Les principes de cohésion et de connexité restent-ils opératoires dans l'agencement prosodique de ces unités ?

Pour aborder ces questions, nous nous inscrivons dans la continuité des travaux développés en macrosyntaxe au GARS ${ }^{\mathrm{ii}}$ (Blanche-Benveniste 1990, Benzitoun et al. 2010, Deulofeu et al. 2010). La perspective macrosyntaxique présente en effet l'avantage de pouvoir représenter tout type de configurations : non seulement celles qui sont agencées autour des marqueurs de rection mais également les relations qui ne peuvent pas être décrites à partir des propriétés rectionnelles des classes grammaticales, comme dans les exemples suivants :

(3) les réformes plus vite et plus fort [Rhap-D2013, Rhapsodie]

(4) il y a plein de trucs tu les vois après en fait les défauts [C-ORAL-ROM]

(5) moi ma famille j'avais que ma mère quand j'habitais là [Corpaix]

Nous posons l'hypothèse que chacun des exemples ci-dessus forme une unité macrosyntaxique de base, c'est-à-dire une unité nécessaire et suffisante pour identifier un et un seul acte illocutoire (voir le principe d'interprétabilité pragmatique dans Cresti et Moneglia 2018). Notre propos est de comprendre comment l'enchaînement de ces unités illocutoires se synchronise avec les phrasés mélodiques dans le discours. Dans cette 
communication, nous nous centrons sur l'unité prosodique majeure, telle qu'elle a été définie sur des bases acoustiques et perceptives dans le modèle de Lacheret-Dujour et Victorri (2002), et dénommée période intonative par les auteurs. L'étude présentée a été effectuée sur le Treebank Rhapsodie ${ }^{\text {iii }}$ (Lacheret-Dujour et al. 2019), un corpus constitué de 57 enregistrements courts de 5 minutes en moyenne; soit 3 heures au total transcrites orthographiquement et phonologiquement, et alignées au son. Ce corpus est échantillonné autour de quatre genres discursifs : argumentatif, descriptif, procédural et oratoire.

L'approche présente trois caractéristiques majeures. D'une part, les unités manipulées sont issues d'un traitement modulaire : les annotations syntaxiques et prosodiques ont été réalisées en parallèle, de façon totalement indépendante et ne se recouvrent donc pas a priori. Ensuite, il s'agit d'une des premières tentatives de modélisation, c'est-à-dire de représentation explicite et quantifiée des différents types d'alignement que l'on peut observer au-delà des chunks, à l'interface de la prosodie, de la syntaxe et du discours (pour des expériences similaires, voir Degand et Simon 2009, Simon et Degand 2011, Martin et al. 2014). Enfin, les variations observées dans les modes de synchronisation des unités illocutoires et des périodes intonatives sont commentées à la lumière des différents genres recensés dans le treebank Rhapsodie. Ce travail nous a conduit notamment à épingler des constructions singulières au regard d'une conception idéalisée des rapports entre prosodie et syntaxe d'une part, prosodie et activité illocutoire d'autre part (discutée dans la section 2). Nous posons l'hypothèse que ces constructions, que nous appelons constructions fragmentées et asynchrones (section 3), sont fonctionnellement et/ou cognitivement interprétables. En effet, un genre appelle ses propres contraintes de production et sélectionne ses propres marques pour instancier la structure communicative dans le message. De-même, la planification discursive qui sous-tend les phrasés mélodiques varie d'une situation de discours à une autre. Ces phénomènes expliquent l'occurrence de telles constructions dans certains types de discours.

\section{Prosodie et syntaxe en français parlé : complémentarité, suppléance, redondance}

S'interroger sur l'interface intonsyntaxique de la parole ordinaire aujourd'hui, c'est d'un côté tenter de comprendre dans quelle mesure et jusqu'à quel point la syntaxe pilote la segmentation prosodique dans la production de la parole et, de l'autre, voir comment les constructions prosodiques permettent d'accéder à la structure syntaxique dans la réception et la compréhension du message en temps réel. Un exposé rapide des problèmes et limites rencontrés dans les approches actuelles, tant formelles, issues de la phonologie multilinéaire, que fonctionnelles, centrées sur la syntaxe des langues parlées et ses fondements pragmatiques $(\S 2.1)$, nous permet de présenter la spécificité de nos unités d'analyse, l'unité illocutoire d'abord, la période intonative ensuite, et les bases qui ont conduit à les définir totalement indépendamment l'une de l'autre (§ 2.2).

\subsection{Question de méthode, ou " pour en finir avec la phrase syntaxique "}

Pour les approches formelles, dont le but est principalement de comprendre le système prosodique d'une langue et ses propriétés typologiques (Nespor 2010), la structure prosodique dérive de contraintes plurielles: syntaxiques, phonologiques, métriques et sémantico-pragmatiques. Dans les approches fonctionnelles centrées sur la compréhension des propriétés syntaxiques de la parole ordinaire, sa variation et ses usages, la prosodie et la syntaxe sont la plupart du temps vues comme des modules collaboratifs, mobilisés indépendamment pour encoder la structure communicationnelle du message et répondre à des contraintes pragmatiques de différents niveaux. Dans les deux approches, la relation 
entre prosodie, syntaxe et structure informationnelle est une question centrale : la syntaxe et la prosodie sont en principe ${ }^{\mathrm{iv}}$ deux modules parallèles qui coopèrent dans des rapports de complémentarité, de suppléance ou de redondance. L'indépendance relative de la prosodie est donc posée comme un postulat de principe.

Néanmoins, dans les modèles formels, la représentation syntaxique constitue toujours le point de départ pour le traitement prosodique (Selkirk 2005). La structure prosodique est donc subordonnée à la structure syntaxique (pour une revue, voir Delais-Roussarie 2005). Des règles dites de "restructuration » sont ensuite mobilisées pour générer la structure prosodique terminale bien formée d'un énoncé. Par ailleurs, dans ce cadre théorique, l'unité intonative maximale au sein de laquelle l'analyse est conduite reste largement sousexplorée et mal définie. Au mieux, il s'agit de l'énoncé phonologique (Nespor et Voegel 1986) dont on nous dit qu'il peut inclure dans sa portée plusieurs phrases syntaxiques sous certaines conditions sémantiques (présence d'ellipses et d'anaphores, relations de discours, etc.). Au pire et le plus souvent, il s'agit ni plus ni moins de la phrase syntaxique dont les spécialistes de la langue parlée ont parfaitement montré à quel point la notion était peu satisfaisante sur le plan définitoire et pas du tout opératoire pour décrire la parole ordinaire (voir entre autres : Berrendonner 1990, Miller \& Weinert 1998, Charolles et al. 2002, Cresti et Moneglia 2005, Raso \& Mello 2014).

C'est pourquoi nombreux sont ceux qui ont proposé, sur les bases d'un traitement autonome de la prosodie, de définir une unité de référence plus appropriée à la réalité de l'oral (voir les concepts de période chez Hazaël-Massieux 1983 et Berrendonner 1990, 2002, 2013, de paragraphe intonatif chez Morel et Danon-Boileau 1998, ou encore de patron illocutif chez Cresti et Moneglia 2011). Le traitement prosodique est dit autonome, car il intervient avant et indépendamment des autres niveaux de traitement (analyses pragmatiques et syntaxiques); il se fonde en premier lieu sur l'identification perceptive par des sujets humains de frontières dites terminales. Mais là encore, même si cela n'est pas formulé explicitement, la finitude sémantico-pragmatique reste une condition de bonne formation des unités prosodiques majeures ; elle en fixe l'étendue et les limites. Autrement dit, la frontière terminale est considérée comme telle, non pas en vertu de propriétés acoustiques stables et clairement répertoriées ${ }^{\mathrm{v}}$ mais parce qu'elle correspond à un énoncé pragmatiquement bien formé, c'est-à-dire un énoncé perçu comme un tout cohésif et autosuffisant pour l'interprétation. Dans une telle approche où il s'agit de postuler une relation bi-univoque entre une forme sonore perçue (frontière terminale) et sa fonction pragmatique (clôture d'un énoncé pragmatiquement autonome), une unité linguistique majeure, quelle qu'en soit la nature (informationnelle, illocutoire) et quel que soit le nom qu'on lui donne, ne peut jamais être fragmentée en unités prosodiques majeures au risque de rompre la cohésion intonodiscursive. L'autonomie prosodique supposée reste une déclaration de principe et une certaine circularité demeure.

Forts de ce constat et en nous fondant sur nos travaux préliminaires conduits depuis 2002 sur l'interface intonosyntaxique et son interprétation fonctionnelle, nous avons adopté une démarche radicalement différente où le statut d'unité prosodique maximale n'est jamais déterminé par des conditions de bonne formation syntaxique et/ou sémantico-pragmatique mais uniquement par ses propriétés sonores : mélodiques et temporelles.

Le traitement intonosyntaxique qui fonde la démarche consiste à appréhender la prosodie et la syntaxe comme des processus dynamiques d'assemblages plus ou moins complexes d'éléments (pour des points de vue précurseurs sur ce thème, voir Auer 2009, Martin 2009), qui reposent sur différents modes de synchronisation temporelle entre les deux niveaux d'organisation du message. Ces assemblages conduisent à l'émergence en temps réel d'unités prosodiques dont il s'agit dans un premier temps d'établir les propriétés formelles, pour en proposer ensuite une lecture fonctionnelle et enfin préciser les variables situationnelles, en particulier les genres de discours, qui sous-tendent ces constructions. 


\subsection{L'unité illocutoire et la période intonative : unités macrosyntaxique et intonative majeure}

L'unité illocutoire (UI) est ainsi désignée car elle est toujours porteuse d'une force illocutoire. Elle se construit autour d'un noyau obligatoire (en gras dans les exemples cidessous) qui porte la force illocutoire proprement dite et possède à ce titre une autonomie illocutoire : le noyau constitue à lui seul un énoncé bien formé. Les ad-noyaux sont des composantes périphériques qui n'ont pas d'autonomie illocutoire et sont donc dans la dépendance illocutoire du noyau. Ils peuvent être antéposés au noyau, on parle alors de prénoyau (ex 6), post-posés, il s'agit de post-noyaux (ex 7) ou intégrés dans les cas des innoyaux $(\mathrm{ex} 8)^{\mathrm{vi}}$.

(6) alors < là < la psychiatrie < c'est autre chose [Rhap-D0006, CFPP2000]

(7) ça a duré dix ans $>$ le silence autour de moi [Rhap-D2001, Corpus Mertens]

(8) c'est une expérience (ça) que je n'ai jamais oubliée [Rhap-D2001, Mertens]

La période intonative quant à elle n'est pas définie en fonction des éléments qui la composent mais à partir des frontières qui la ponctuent. Ces frontières sont calculées sur les bases de variations acoustiques perceptivement significatives et entraînant une réinitialisation de la structure prosodique (Fig.1).

A cette étape de l'analyse, nous ne savons rien sur la fonction de ces frontières. Nous pouvons seulement supposer qu'elles viennent clore un segment qui a priori joue un rôle essentiel pour la compréhension du message. En d'autres termes, tout élément qui doit donner lieu à un traitement cognitif élaboré serait associé à une frontière prosodique (Chafe $1998)^{\text {vii }}$. Le statut pragmatique de cet élément (fonction textuelle, communicationnelle, illocutoire, expressive) est une autre question, particulièrement complexe, qui repose sur la prise en compte d'un ensemble de paramètres, relevant de ses propriétés internes d'une part (intonatives, syntactico-sémantiques et lexicales), de sa distribution dans l'énoncé d'autre part et, enfin, du contexte. Pour résumer la frontière prosodique terminale considérée indépendamment du matériel verbal est polyfonctionnelle.

$\mathrm{Du}$ point de vue de l'implémentation phonétique, le traitement réalisé semiautomatiquement (annotation automatique nettoyée manuellement) avec le logiciel Analor $^{\text {viii }}$ est fondé sur l'hypothèse que la perception d'une frontière n'est pas seulement basée sur l'occurrence, la forme et l'amplitude de contours intonatifs, mais sur la combinaison de trois marqueurs acoustiques et de leur force relative : une pause d'une certaine durée, un contour intonatif ample avant la pause, dont la fréquence terminale se situe au niveau suraigu ou infra-grave, et une réinitialisation mélodique après la pause. Ainsi, dans la figure 1, la frontière de période intonative à la fin de la séquence je tombe sur la place Sainte-Claire on va dire là où il y la halle est marquée simultanément par un continuatif majeur, une pause de plus de $400 \mathrm{~ms}$ et une réinitialisation mélodique dans le niveau moyen qui permet de repartir sur une nouvelle séquence et $j$ 'arrive sur la place Notre Dame.

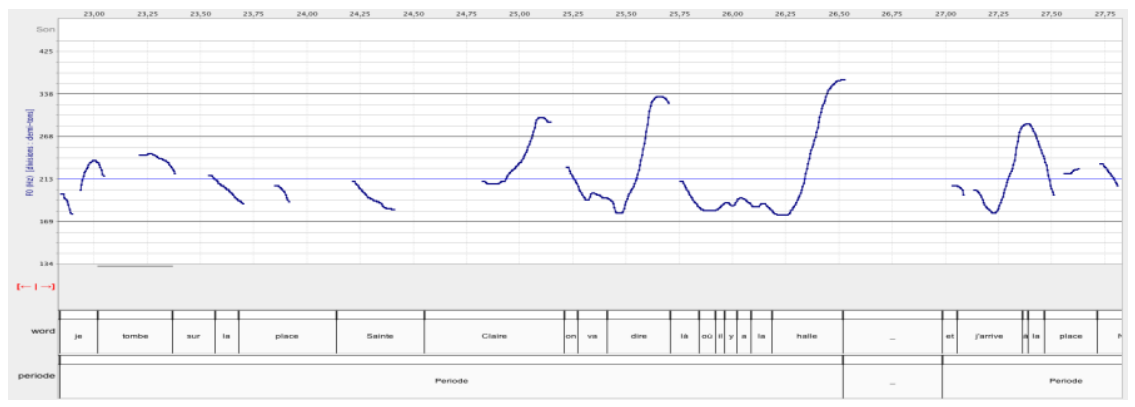


Fig. 1. Exemple de segmentation en deux périodes intonatives de la séquence je tombe sur la place Sainte-Claire on va dire là où il y a la halle \# et j'arrive à la place Notre-Dame [Rhap-M0014, Avanzi].

\section{Périodes intonatives et unités illocutoires terminales dans le treebank Rhapsodie}

Après avoir présenté une cartographie d'ensemble (§ 3.1) des frontières d'unités illocutoires (FUI) et de périodes intonatives (FPI), nous discutons de leurs combinaisons dans les échantillons étudiés ( $\$ 3.2$ ). Pour finir, nous précisons leurs propriétés formelles et proposons une lecture fonctionnelle des variations observées au regard des genres de discours dans lesquelles elles s'inscrivent (§ 3.3).

\subsection{Cartographie générale}

Rhapsodie contient 2849 périodes intonatives et 3154 unités illocutoires, soit un ratio général FUI/FPI de 1.10. Si ce ratio révèle plus de constructions inclusives que fragmentées, c'est-à-dire plus de périodes intonatives qui fusionnent plusieurs unités illocutoires, que d'unités illocutoires segmentées sur plusieurs périodes intonatives, il ne dit rien sur la distribution précise de ces frontières et les différents types de combinaisons susceptibles d'être rencontrés dans le treebank. Une observation plus fine montre que la distribution peut varier significativement d'un échantillon à l'autre (figure 2). Alors que certains échantillons ont un ratio FUI/FPI de 5 ( 5 frontières d'unités illocutoires pour 1 frontière de période intonative), d'autres ont une distribution diamétralement opposée (5 frontières de périodes intonatives pour 1 frontière d'unité illocutoire). Autrement dit, pour un nombre fixe de FPI, la proportion de FUI peut varier de 1 à 25 !

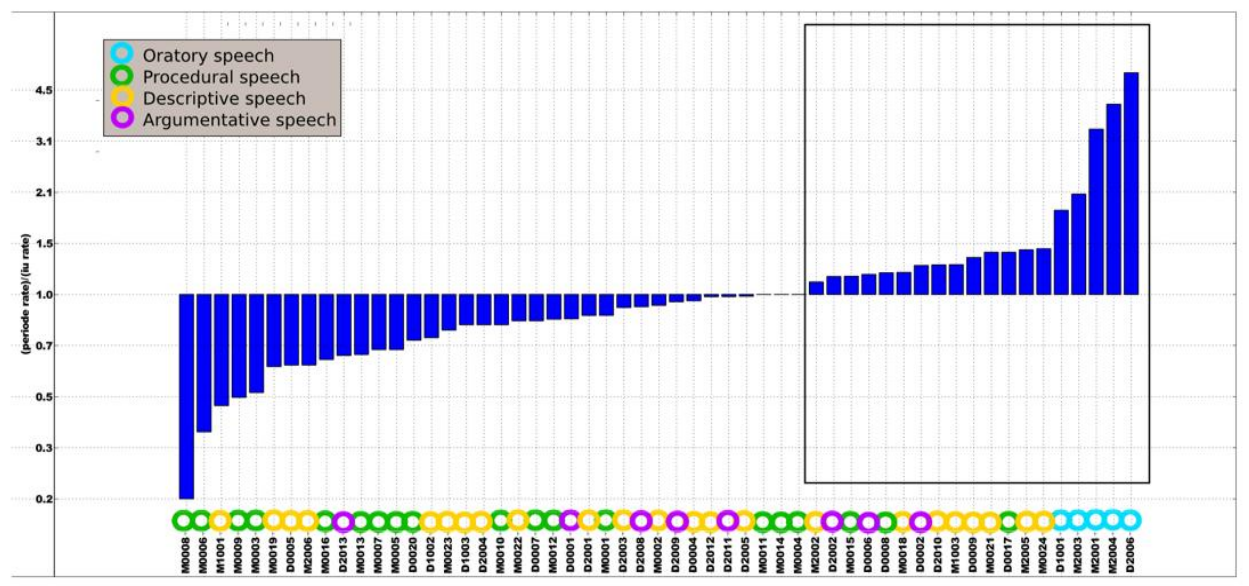

Fig. 2. D'après Beliao (2016), représentation logarithmique des ratios FPI/FUI par échantillon, de gauche à droite des échantillons qui contiennent le plus de FUI aux échantillons qui ont le plus de FPI.

\subsection{Les frontières et leurs combinaisons}

On comprend d'après la figure 2 que le nombre équivalent de périodes intonatives et d'unités illocutoires dans le Treebank Rhapsodie n'implique pas que leurs frontières soient 
globalement synchronisées. Ainsi, (9) illustre trois types d'appariement sur le même flux temporel : une frontière prosodique non synchronisée avec une frontière d'unité illocutoire, une frontière d'unité illocutoire non synchronisée avec une frontière prosodique, enfin deux frontières synchronisées. Le tableau 1 donne une vue d'ensemble des différentes combinaisons de frontières ( $41 \%$ de frontières synchronisées vs. $59 \%$ non synchronisées).

(9) tu continues la rue FPI la petite rue FUI et tu arrives à la fontaine euh place NotreDame FPI-FUI [Rhap-M000, Avanzi]

Table 1. Alignements entre frontières : fréquences et ratios

\begin{tabular}{|l|l|l|}
\cline { 2 - 3 } \multicolumn{1}{c|}{} & Fréquence & $\%$ \\
\hline FPI-FUI & 1742 & 41 \\
\hline FPI non synchronisées & 1412 & 33 \\
\hline FUI non synchronisées & 1107 & 26 \\
\hline Total des frontières & 4261 & 100 \\
\hline
\end{tabular}

La figure 3 représente le pourcentage de synchronisation des FPI (axe des ordonnées) et des FUI (axe des abscisses) pour chacun des 57 échantillons Rhapsodie. Dans les extraits situés sur le bord droit du diagramme, les FUI sont parfaitement synchronisées avec les FPI ; pour les extraits situés en bordure supérieure, les FPI sont toujours synchronisées avec les FUI. Soit, pour préciser notre propos, les échantillons [Rhap-M2004, Rhapsodie], [Rhap-M0008, Avanzi], [Rhap-M0014, Avanzi]. Dans le premier, marqué par un nombre significativement plus important de FPI que de FUI, l'occurrence d'une frontière d'unité illocutoire est toujours couplée à la production d'une frontière de période intonative. Pour le second, où ce sont les FUI qui dominent, les FPI sont synchronisées. Enfin, le dernier ne contient que des frontières synchronisées. Quant aux échantillons sur la diagonale, de bas en haut en partant de la gauche, s'ils ont tous un ratio FPI/FUI de 1, cela ne suppose pas que leurs frontières soient synchronisées. En pratique, plus la distance entre l'échantillon et le bord supérieur droit du diagramme est importante moins les frontières sont synchronisées. Ainsi, [Rhap-M0022, Rhapsodie] est produit par une alternance régulière de FPI et de FUI, comme en témoigne (10) extrait de l'échantillon, où nous avons plusieurs UI syntaxiquement enchâssées regroupées dans une même période.

(10) donc il embarque euh mh le Chaplin FPI pour ensuite on se retrouve FUI donc tout le monde s'en va y compris la foule FPI puisque la foule est intéressée par les flics euh FUI on se retrouve donc avec le boulanger FPI la jeune fille au sol et la vieille dame qui revient vers le boulanger pour lui dire non FUI ce n'est pas le monsieur FUI mais c'est la jeune fille effectivement FPI-FUI [Rhap-M0022, Rhapsodie] 


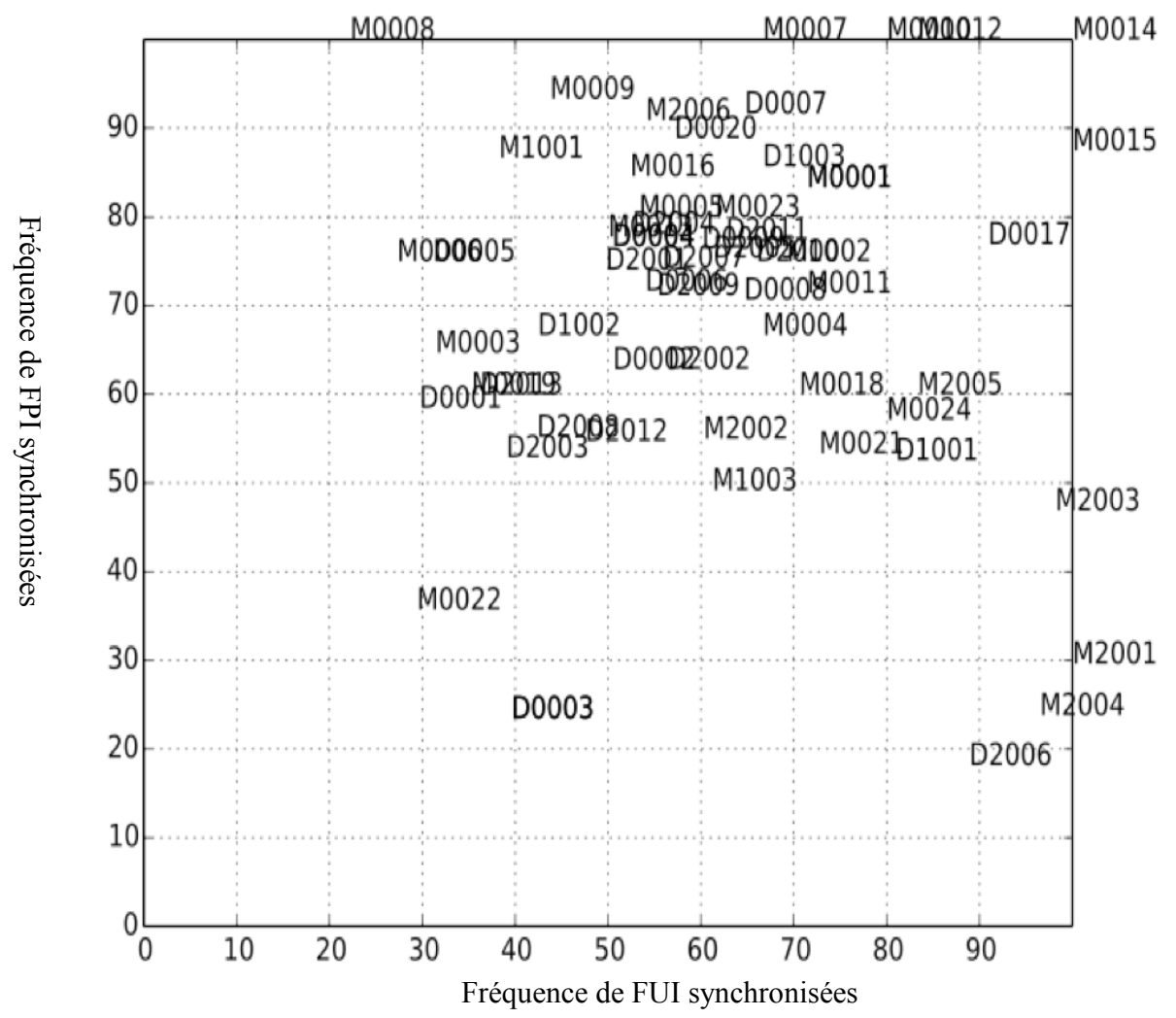

Fig. 3. D’après Beliao (2016), proportion des FPI et des FUI synchronisées par échantillon

En écho à la figure 2, nous observons que les discours oratoires (ex. [Rhap-M2004, Rhapsodie], [Rhap-M2003, Rhapsodie], [Rhap-M2001, C-Prom], [Rhap-D2006, Rhapsodie]) sont caractérisés par un nombre très important de FPI non synchronisées. A l'inverse, la synchronisation des FPI avec les FUI semble constituer une propriété formelle de la parole procédurale (ex. [Rhap-M0007, Avanzi], [Rhap-M0007, Avanzi], [Rhap-M0008, Avanzi], [Rhap-M0015, Avanzi]). Reste à comprendre les différents types d'enchaînement des frontières, particulièrement dans les contextes de non-synchronisation et leurs corrélations avec les genres de discours.

\subsection{Distribution en enchaînement des frontières : propriétés formelles et interprétation fonctionnelle}

Sachant que leur distribution n'est pas uniforme, nous nous sommes particulièrement intéressés aux frontières non synchronisées et à la façon dont elles se répartissent sur un segment de parole situé entre deux frontières synchronisées. Nous sommes partis de l'hypothèse que les frontières synchronisées sont des points cruciaux pour la réinitialisation des flux syntaxique et prosodique et que, par conséquent, les segments produits entre deux frontières de ce type ont une cohésion discursive interne dont les constructions intonosyntaxiques devraient rendre compte. A ce titre, nous les avons appelés unités intonosyntaxiques (UIS). Pour effectuer ce travail, nous avons extrait aléatoirement 388 segments du corpus Rhapsodie qui se répartissent en quatre types d'UIS dont la fréquence d'occurrence est fournie dans le tableau 2. 
Table 2. Distribution des frontières et types d'UIS

\begin{tabular}{|l|l|}
\hline Distribution & Rà \\
\hline Aucune frontière interne & $42 \%$ \\
\hline Une frontière interne non synchronisée & $22 \%$ \\
\hline Au moins deux frontières internes non synchronisées & $36 \%$ \\
\hline Types & \\
\hline UIS synchronisée & $42 \%$ \\
\hline UIS inclusive & $25 \%$ \\
\hline UIS fragmentée & $21 \%$ \\
\hline UIS asynchrone & $12 \%$ \\
\hline
\end{tabular}

L'UIS synchronisée ne contient ni FUI ni FPI interne comme dans l'exemple 11 construit autour de deux UIS synchronisées. Cette configuration est illustrée par la figure 4, où l'unité illocutoire est représentée par une boîte blanche et la période intonative par une boîte noire.

(11) après tu montes une grande ligne droite FPI-FUI tu passes devant la piscine FPI-FUI [Rhap-M0009, Avanzi]

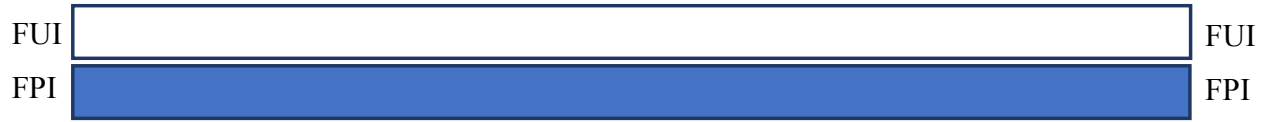

Fig. 4. UIS synchronisée : une unité illocutoire coïncide avec une période intonative

Parmi les segments non synchronisés, nous trouvons des segments articulés autour d'une ou plusieurs FUI. Autrement dit, dans ce type de segment, la période intonative inclut dans sa portée une ou plusieurs unités illocutoires; on parle donc de construction inclusive (ex 12 et figure 5).

A l'inverse, les segments structurés autour d'une ou plusieurs FPI fragmentent une unité illocutoire en plusieurs périodes intonatives (ex 14, figure 6).

Enfin, les segments asynchrones, qui contiennent à la fois des FPI et des FUI et pour lesquels il y a donc chevauchement de frontières, se construisent simultanément autour de la fragmentation et de l'inclusion (ex 14, figure 7).

(12) euh il y a un stade aussi à côté FUI et tu arrives à un rond-point FUI donc là tu rentres dans le centre-ville FPI-FUI [Rhap-M0009, Avanzi]

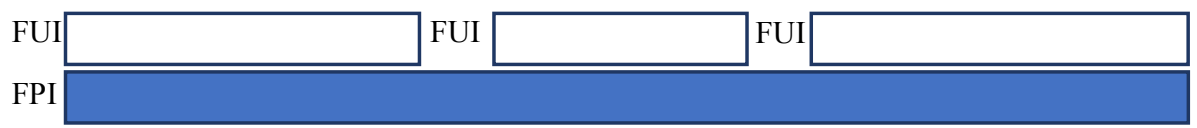

FUI FPI

Fig. 5. UIS inclusive : une période intonative contient trois unités illocutoires

(13) je pense aux nombreuses victimes de la tempête FPI et à toutes les familles FPI endeuillées FPI dont nous partageons la peine FPI-FUI [Rhap-M2004, Rhapsodie]

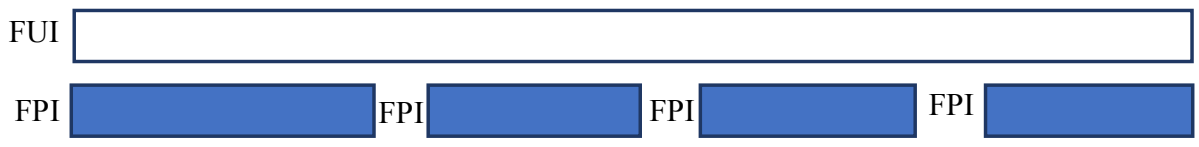

Fig. 6. UIS fragmentée : une unité illocutoire contient quatre périodes intonatives 
(14) mais à cette époque FPI on vous remarquerait FUI et d'ailleurs quelqu'un vous a remarquée qui est dans une grande limousine noire FPI-FUI [Rhap-2010, Rhapsodie]

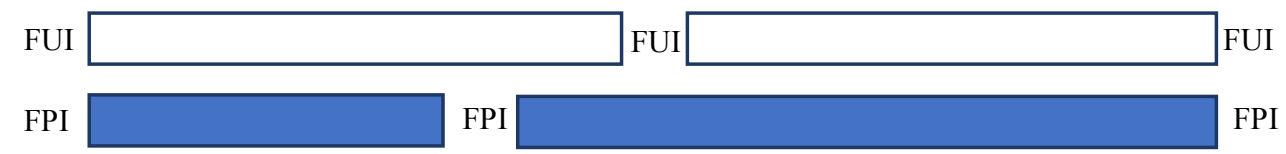

Fig. 7. UIS asynchrone : une unité illocutoire et une période intonative se chevauchent sans se synchroniser

D'un point de vue discursif, fonctionnel, l'UIS inclusive est caractéristique du discours procédural, on l'a vu (figure 3), et est aussi très fréquemment produite dans la parole descriptive, quel que soit l'objet de la description. Une telle construction permet de grouper une succession de faits discursivement liés dans un même ensemble; elle donne à voir les différentes étapes de la progression thématique. Ainsi, dans l'exemple 15, les trois premières unités illocutoires instancient un seul objet de discours (l'énonciateur lui-même) et sont à ce titre regroupées dans une période intonative unique. La production d'une nouvelle période intonative permet d'enchaîner sur un nouvel objet de discours (le diplôme du bac).

(15) alors euh je m'appelle Clara FUI j'ai dix-neuf ans FUI j'ai eu l'obtention de mon bac euh donc l'année dernière FPI-FUI c'est un bac euh SMS donc technologique FPI-FUI Rhaps-M1001, Rhapsodie]

Dans des descriptions d'itinéraires (14 échantillons dans le corpus Rhapsodie), ces faits sont essentiellement des séquences d'actions (Lacheret-Dujour et al. 2007) qui décrivent les étapes élémentaires d'un trajet global (ex 16).

(16) euh il y a un stade aussi à côté FUI et tu arrives à un rond-point FUI donc là tu rentres dans le centre-ville FPI-FUI [...] après là c'est indiqué FUI il y a la une cabine de téléphone FUI et voilà c'est là FPI-FUI [Rhaps-M009, Avanzi]

Enfin, cette fonction de liage discursif peut être couplée à des phénomènes de reformulation et de correction où il s'agit de préciser le propos (ex 17 et 18).

(17) et au bout d'un temps vous allez arriver sur une grande place avec une fontaine au milieu FUI c'est la place Victor Hugo FPI-FUI [Rhaps-M0013, Avanzi]

(18) et euh je reprends je crois le non c'est pas le boulevard Gambetta FUI je ne sais pas lequel c'est euh FUI c'est l'autre FPI-FUI [Rhaps-M0016, Avanzi]

L'UIS fragmentée, représentative du discours oratoire, y a essentiellement pour fonction d'indiquer des parallélismes énonciatifs, construits autour de listes paradigmatiques (voir Kahane et Pietrandrea 2012 pour une présentation exhaustive de ce type de constructions et des valeurs sémantiques et discursives qu'on peut lui attribuer), que nous assimilons ici à des opérations de focalisation réalisées par un dépliage progressif du propos (ex 19) ${ }^{\mathrm{ix}}$.

(19) elle salue le courage FPI elle salue la loyauté FPI elle salue le dévouement FPI elle salue l'esprit de sacrifice FPI de ceux qui ont choisi de servir sa défense FPI ses intérêts FPI et ses valeurs FPI à travers le monde FPI-FUI [Rhap-M2001, C-Prom] 
Quand elle s'articule autour du thème et $\mathrm{du}$ propos, cette construction par décondensation permet de poser progressivement le ou les cadre(s) du discours, le thème puis le propos (ex 20).

(20) le quatorze juillet FPI lors du traditionnel défilé FPI c'est la nation toute entière FPI qui vous rend hommage FPI-FUI [Rhap-M2001, C-Prom]

Lorsqu'elle est convoquée dans d'autres genres de discours, la construction fragmentée a pour effet de proposer une lecture segmentée (Avanzi 2010) de deux unités dont la seconde est syntaxiquement liée à la première. Elle constitue une instruction interprétative qui conduit l'auditeur à considérer la séquence comme sémantiquement autonome malgré son statut syntaxiquement intégré. Ainsi, la discontinuité de la linéarité discursive induite par le découpage prosodique dans (21) et (22) mène à une interprétation prédicative de la relative (voir le concept de prédication seconde dans Cadiot et Furukawa 2000).

(21) et je passe sous le lycée Stendhal un petit passage FPI qui m'amène vers le square des postes FPI-FUI [Rhap-M0011, Avanzi]

(22) et je euh je continue je continue jusqu'à une place qui est face à la grande poste FPI où il y a la euh Chambre de Commerce et d'Industrie FPI-FUI [Rhap-M0016, Avanzi]

Le taux faible de l'UIS asynchrone tend à montrer que les locuteurs évitent de produire en alternance des FPI et des FUI, c'est-à-dire des chevauchements entre unités prosodiques et macrosyntaxiques majeures. Quand elle est présente, cette unité témoigne surtout de tâtonnements énonciatifs et de difficultés de planification textuelle. Parmi les causes de ces tâtonnements que l'on peut invoquer à la lumière des données observées, on note dans certains échantillons, notamment en situation de lecture, un maillage complexe entre le discours courant et le discours rapporté, (ex 23) ; dans d'autres, en particulier le discours narratif, c'est la réactivation difficile de faits anciens et pas directement accessibles à la mémoire, peut-être approximatifs, qui expliquent que l'énonciateur a du mal à prendre en charge le contenu propositionnel de l'énoncé (ex 24).

(23) Laurent Joffrin parle lui aussi de désaveu cinglant même si dit-il les responsables de l'UMP FPI s'obstinent à nier l'évidence dans un accès de mauvaise foi soviétique et dans un festival de langue de bois FUI l'UMP FPI voulait croire à la bourrasque FUI c'est une tornade FPI-FUI [Rhap-D2013, Rhapsodie] ${ }^{\mathrm{x}}$

(24) je suis née à Cannes FPI pendant la guerre FUI à la fin de la guerre de dix-huit FPI mon père était au front FUI et nous avons été chassés d'A $\sim$ d'Alsace naturellement FPIFUI [Rhap-D2004, Lacheret]

\section{Conclusion}

Comment la syntaxe et la prosodie collaborent pour façonner en temps réel le discours dans la parole de tous les jours reste une question majeure pour comprendre et représenter l'interface intonosyntaxique dans les langues parlées, sa structuration et ses fonctions discursives. Nous avons abordé cette question en nous focalisant sur les unités syntaxiques et prosodiques majeures qui restent encore très difficiles à identifier et donc sous-explorées en linguistique. Nous avons rappelé les critères utilisés pour définir ces unités, que nous avons appelées respectivement unités illocutoires et périodes intonatives, et les identifier dans la chaîne parlée. Nous avons ensuite observé les différents modes de synchronisation 
de leurs frontières terminales respectives telles qu'elles ont été identifiées dans le treebank Rhapsodie, un corpus multigenre, constitué de 57 échantillons (argumentatifs, descriptifs, procéduraux et oratoires) de 5 minutes en moyenne, et offrant 3 heures d'enregistrement transcrites orthographiquement, phonologiquement, et alignées au son. Nous avons pu voir que si $42 \%$ de frontières sont parfaitement synchronisées et témoignent donc d'un alignement des unités, 58\% relèvent d'un autre mode d'appariement. En pratique, deux constructions ont été régulièrement observées : la construction par inclusion, lorsqu'une période intonative fusionne plusieurs unités illocutoires, et la construction par fragmentation quand une unité illocutoire est segmentée en plusieurs périodes intonatives. Nous avons montré comment les différents types de phrasés intonatifs majeurs, en proposant une lecture intégrée ou segmentée des unités syntaxiques, guident leur traitement sémantico-pragmatique. Cette situation conduit à interroger à nouveau et sur de nouvelles bases les règles de connexité et de cohésion syntaxique postulées comme allant de soi par les chercheurs.

Nous avons mis en regard ces constructions avec les différents genres de discours présents dans Rhapsodie et montré comment la prosodie est mobilisée de façon variable dans les genres pour structurer le flux discursif et actualiser la structure communicative dans le message (cadrage discursif, détachement thématique, focalisation, etc.). Plus rarement, nous avons également épinglé une construction par chevauchement qui contient simultanément des frontières prosodiques et syntaxiques indépendantes les unes des autres. La proportion faible de ce dernier mode de synchronisation montre que les locuteurs évitent de produire une alternance de frontières de nature différente entre deux frontières synchronisées. Ce type de combinaison semble découler de la structure de certains échantillons comme ceux qui alternent discours courant et discours rapporté, mais également de certaines conditions matérielles très particulières lors de la prise de parole (temps de parole compté dans les journaux radiophoniques lus) qui entraînent des modes de planification singuliers. Les constructions par chevauchement témoignent également de la complexité de la tâche caractéristique de la parole improvisée, où il s'agit de conceptualiser et de produire simultanément un discours.

Sous l'angle de la linguistique de corpus, ce premier travail nous a permis de fixer une méthodologie pour tester à grande échelle le rendement fonctionnel des constructions observées et généraliser nos hypothèses. Du point de vue de la linguistique expérimentale et de la psycholinguistique, nous avons là des outils pour sonder plus avant le rôle et la pertinence des différents modes de synchronisation intonosyntaxiques observés pour l'interprétation et le traitement en temps réel du message parlé.

\section{Références bibliographiques}

Abney, S. P. (1991). Parsing by chunks. Principle-based Parsing: Computation and Psycholinguistics $44,257-278$.

Auer, P. (2009). On-line syntax: Thoughts on the temporality of spoken language. Language Sciences, 31(1), 1-13.

Avanzi, M. (2010). Rattachement et fragmentation de la syntaxe par la prosodie. Travaux de linguistique, (1), 145-166.

Beliao, J. (2016). How Prosody and syntax are mapping : a study of synchronization and congruence, Doctorat en Sciences du langage, Paris Nanterre.

Benzitoun, C., Dister, A., Gerdes, K., Kahane, S., Pietrandrea, P. et Sabio, F. (2010). Tu veux couper là faut dire pourquoi : propositions pour une segmentation syntaxique du français parlé. Actes CMLF 2010, 2075-2090. 
Berrendonner, A. (1990). Pour une macro-syntaxe. Travaux de linguistique 21, 25-31.

Berrendonner, A. (2002). Les deux syntaxes. Verbum, (1-2), Presses universitaires de Nancy, 23-35.

Berrendonner, A. (2013 ed.). Grammaire de la période, Bern : Peter Lang.

Blanche-Benveniste, Cl. (1990 éd.). Le français parlé. Paris : Editions du CNRS.

Cadiot, P. et Furukawa N. (2000). La prédication seconde. Langue Française, 127.

Chafe, W. (1998). Language and the Flow of Thought. The New Psychology of Language, M. Tomasello (éd.), New Jersey, Lawrence Erlbaum Publishers, 93-111.

Charolles, M., Le Goffic, P., Morel, M. A. (2002 éds). Y at-il une syntaxe au-delà de la phrase? Verbum (1.2), Presses universitaires de Nancy.

Cresti, E. et Moneglia, M. (2005 eds). C-ORAL-ROM. Integrated Reference Corpora for Spoken Romance Languages. Amsterdam : John Benjamins.

Cresti, E. et Moneglia, M. (2011). Annotation de corpus selon la théorie de la langue en actes. Langue française 170, 95-110.

Cresti, E., et Moneglia, M. (2018). The illocutionary basis of information structure. Information Structure in Lesserdescribed Languages, 359-402.

Degand L. et Simon, A.C. (2009). Minimal discourse units in spoken French: On the role of syntactic and prosodic units in discourse segmentation. Discours4. Retrieved from: discours. revues. $\operatorname{org} / 5852$.

Delais-Roussarie, E. (2005). Phonologie et Grammaire : Etudes et modélisation des interfaces prosodiques. Mémoire d'Habilitation à Diriger des Recherches, Université de Toulouse-le Mirail.

Deulofeu, J., Gerdes, K., Kahane S. et Pietrandrea, P. (2010). Depends on what the French say: Spoken corpus annotation with and beyond syntactic function. Proceedings of $L A W I V, A C L$, 274-281.

Di Cristo, A. (1975). Recherches sur la structuration prosodique de la phrase française. Actes des 6èmes journées d'étude sur la parole, 95-116.

Hazaël-Massieux, M. C. (1983). Le rôle de l'intonation dans la définition et la structuration de l'unité de discours. Bulletin de la Société de Linguistique de Paris, 78, 99-160.

Kahane, S. et Pietrandrea, P. (2012). Types d'entassement en français. Actes du Congrès Mondial de Linguistique Française (CMLF 2012), 1809-1828.

Lacheret-Dujour, A. et Victorri B. (2002). La période intonative comme unité d'analyse pour l'étude du français parlé : modélisation prosodique et enjeux linguistiques. Verbum, 24/1-2, 55-72.

Lacheret-Dujour, A., Victorri, B., et Avanzi, M. (2007). La mise en scène intonative dans la description d'itinéraires en milieu urbain. TRANEL. Travaux Neuchâtelois de Linguistique, (47), 79-102.

Lacheret-Dujour, A, Kahane, S. et Pietrandrea, P. (2019). Rhapsodie, a prosodic and syntactic treebank of spoken French. Amsterdam : John Benjamins.

Martin, P. (1981). Pour une théorie de l'intonation. L'intonation est-elle congruente à la syntaxe ? In L'intonation de l'acoustique à la sémantique, M. Rossi (éd.), Paris, Klincksieck.

Martin (1987). Prosodic and rhythmic structure in French. Linguistics, 25, Mouton de Gruyter, Amsterdam, 925-949.

Martin, P. (2009). Intonation du français. Paris : Armand Colin.

Martin, L., Degand, L. et Simon, A.C. (2014). Forme et fonction de la périphérie gauche dans un corpus oral multigenres annoté. Corpus 13, 243-265.

Mertens, P. (1993). Accentuation, intonation et morphosyntaxe. Travaux de Linguistique 26, 21-69. 
Mertens, P. (1997). De la chaîne linéaire à la séquence de tons. Traitement Automatique des Langues $38(1), 27-51$.

Mertens, P. (2008). Syntaxe, prosodie et structure informationnelle : une approche prédictive pour l'analyse de l'intonation dans le discours. Travaux de linguistique, (1), 97-124.

Miller, J. et Weinert, R. 1998[2009]. Spontaneous Spoken Language. Syntax and Discourse. Oxford: Oxford University Press.

Morel, M. A. et Danon-Boileau, L. (1998). Grammaire de l'intonation l'exemple du français. Paris: Ophrys.

Nespor, M. (2010). Prosody: An interview with Marina Nespor. ReVEL 18(15), 381-387.

Nespor, M. et Vogel, I. (1986). Prosodic Phonology, Dordrecht: Foris.

Selkirk, E. (2005). Comments on intonational phrasing in English. Prosodies: With special reference to Iberian languages, 9, 11-58.

Simon, A.C. et Degand L. (2011). L'analyse en unités discursives de base : pourquoi et comment ? Langue française 170, 45-60.

Raso, T. et Mello, H. (2014). Spoken Corpora and Linguistic Studies, Amsterdam: Benjamins.

Rossi, M. (1981). L'intonation n'est pas congruente à la syntaxe : une explication. In L'intonation de l'acoustique à la sémantique, M. Rossi (éd.), Paris : Klincksieck, 290-296.

i Il n'existe pas de connexion explicite entre ces éléments qui se retrouvent donc isolés dans un ensemble qu'ils contribuent pourtant à constituer comme un tout cohésif.

${ }^{\text {ii }}$ Groupe aixois de recherche en syntaxe.

iii $h$ ttps://www.projet-rhapsodie.fr/

iv La théorie de la langue en actes développée par E. Cresti et son équipe à Florence propose une méthodologie particulière où l'analyse syntaxique repose sur les traitements prosodiques et pragmatiques qui ont été effectués préalablement.

${ }^{v}$ Même si les corrélats acoustiques de ces frontières peuvent être discutés par les auteurs, les descriptions proposées restent globalement très empiriques, variables, d'une approche à une autre et donc difficilement généralisables dans une perspective de modélisation.

${ }^{\text {vi }}$ Le symbole '<' clôt un pré-noyau, '>' introduit un post-noyau; le in-noyau est indiqué entre parenthèses.

${ }^{\text {vii }}$ Voir chez l'auteur, leprincipe de "spotlight of consciousness", selon lequel il faut diriger l'attention de l'interlocuteur sur l'élément essentiel d'un message; en d'autres termes, porter cet élément à sa conscience immédiate.

viii http://www.lattice.cnrs.fr/ressources/logiciels/analor/

ix Le segment "de ceux qui ont choisi de servir sa défense" dépend simultanément de "courage", "loyauté", "dévouement" et "esprit de sacrifice" ; il y a donc une seule unité macrosyntaxique : "elle salue le courage elle salue la loyauté (...) de ceux qui ont choisi de servir sa défense".

${ }^{\mathrm{x}}$ Les frontières de périodes après le sujet, qui donnent lieu ici à la production d'UIS asynchrones, si elles sont syntaxiquement contre-intuitives, n'en sont pas moins rares dans le teebank. Reste à comprendre les mécanismes pragmatiques (encodage de la structure informationnelle); cognitifs (planification, désignation, reformulation) et/ou rythmiques (équilibre des phrasés) qui les motivent. 